\title{
Nanostructured core single mode phosphate fiber laser with high slope efficiency (Conference Presentation) (Withdrawal Notice)
}

Marcin Franczyk, Ryszard Stepien, Adam Filipkowski, Dariusz Pysz, Ryszard Buczyński

Marcin Franczyk, Ryszard Stepien, Adam Filipkowski, Dariusz Pysz, Ryszard Buczyński, "Nanostructured core single mode phosphate fiber laser with high slope efficiency (Conference Presentation) (Withdrawal Notice)," Proc. SPIE 10681, Micro-Structured and Specialty Optical Fibres V, 106810W (18 July 2019); doi: 10.1117/12.2313134

SPIE. Event: SPIE Photonics Europe, 2018, Strasbourg, France 


\section{Nanostruc tured core single mode phosphate fiber laser with high slope efficiency (Conference Presentation) (Withdrawal Notice) \\ Marc in Franczyk, Ryszard Stepien, Adam Flipkowski, Daniusz Pysz, and Ryszard Buczyński \\ Institute of Electronic Materials Technology (Poland)}

Proceedings Volume 10681, Mic ro-Structured and Speciality Optic al Fibres V; 106810W (2018)

https://doi.org/10.1117/12.2313134

Event Micro-Structured and Specialty Optic al Fibres V, 2018, Strasbourg, France

Online Publication Date: 23 May 2018

Withdrawn from Publication: 18 J uly 2019

Publisher's Note: This conference presentation, origina lly published on 23 May 2018, was withdrawn per author request. 